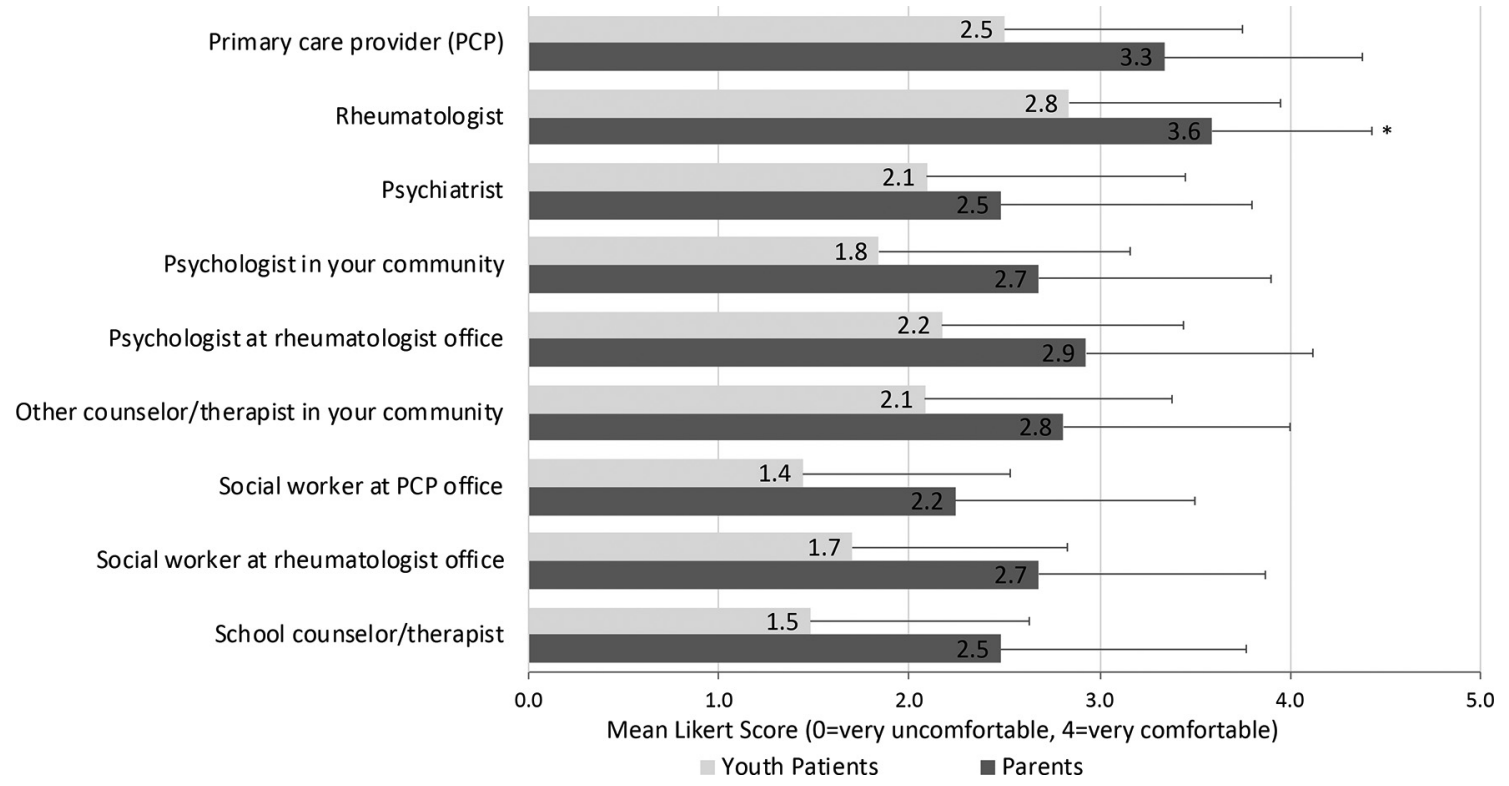

Abstract 92 Figure 1 Comparison of level of comfort with potential mental health providers among youth patients with SLE and parents. Results are shown for linear regression models comparing Likert scores among youth patients and parents, adjusted for disease duration and patient/parentreported visual assessment score for disease-related health. ${ }^{*}=p<0.05,{ }^{* *}=p<0.01,{ }^{* * *}=p<0.001$

youth and parent responses using regression models to examine comfort level with potential mental health providers.

Results 102 respondents included 59 patients (58\%) and 43 (42\%) parents. Youth had a mean age of 20.9 (standard deviation, $\mathrm{SD}=3.4$ ) years, and mean disease duration of 6.9 (SD 4.0) years. History of a mental health problem was reported by 21 youth (36\%), of which $66 \%$ said that their rheumatologist was unaware. Clinician-diagnosed anxiety was reported by $19 \%$, depression by $12 \%$, and adjustment disorders by $19 \%$; another $17 \%, 8 \%$ and $10 \%$ had self-reported symptoms of these disorders, respectively. Mean Likert ratings by youth indicated that disease aspects most impacting mental health were worry about disease impact on the future at 3.0 (SD 1.2), worry about having a flare at 2.9 (1.2), and worry about medication side effects at 2.8 (1.3). Youth and parents felt most comfortable discussing mental health concerns with rheumatologists and primary care providers, and least comfortable with social workers and school counselors (figure 1).

Conclusions Youth with SLE have high rates of diagnosed and undiagnosed mental health problems, which are impacted by their disease. Mental health intervention strategies in rheumatology settings may improve mental health education, screening and treatment for these youth.

Funding Source(s): The Childhood Arthritis and Rheumatology Research Alliance

\section{NEUROPSYCHOLOGICAL FACTORS AND ANTI- NEURONAL ANTIBODIES AS DAMAGE ACCRUAL PREDICTORS IN SYSTEMIC LUPUS ERYTHEMATOSUS}

\footnotetext{
${ }^{1}$ Milena Mimica, ${ }^{2}$ Ignacio Barra, ${ }^{2}$ Rocío Ormeño, ${ }^{2}$ Patricia Flores, ${ }^{2}$ Jorge Calderón, ${ }^{2}$ Oslando Padilla, ${ }^{1}$ Marcela Bravo-Zehnder, ${ }^{1}$ Loreto Massardo, ${ }^{3}$ Alfonso González. ${ }^{1}$ Centro de Biología Celular y Biomedicina (CEBICEM). Faculty of Science and Medicine, Universidad San Sebastián. Santiago, Chile; ${ }^{2}$ Faculty of Medicine, Pontificia Universidad Católica de Chile. Santiago, Chile; ${ }^{3}$ Centro de Biología Celular y Biomedicina. Faculty of Science and Medicine, Universidad San Sebastián. Santiago, Chile. Centro de Envejecimiento y Regeneración (CARE), Facultad de Ciencias, Pontificia Universidad Católica de Chile. Santiago, Chile
}

10.1136/lupus-2019-Ism.93
Background Organ damage may accumulate throughout life. The role of neuropsychiatric lupus manifestations on damage remains unknown. Cognitive dysfunction is present in $17 \%-66 \%$ of patients and an impact on damage accrual is suggested.We found a cognitive deficit association with the presence of anti-dsDNA/methyl-d-aspartate receptor (antidsDNA/NMDAR) and anti-ribosomal $\mathrm{P}$ proteins (anti-P), antibodies that have fluctuating titers with disease activity. Anti-P exerts neuropathogenic potential through recognition of neuronal surface antigens distributed in regions in memory, cognition and emotion. Anti-neuronal surface $\mathrm{P}$ antigen (NSPA), the anti-P target, is involved in neuronal excitatory transmission and mediates anti-P effects on memory dysfunction in mice models

We investigated predictors associated to damage in a SLE cohort with a thorough neuropsychological and antineuronal antibodies baseline assessment, after 4.8 years of follow-up. We include data on a new class of antibodies recognizing NSPA and lacking anti-P reactivity.

Methods From a study on 118 female SLE patients between 2008 and 2013 a subset of 99 SLE patients was followed-up in 2016. Baseline assessment: age, disease duration, therapies and antiphospholipid syndrome (APS). Neuropsychological evaluation: depression (MINI-plus) and cognitive deficit (Cambridge Neuropsychological Test Automated Battery). Antibodies: anti-dsDNA, anti-dsDNA/N-methyl-d-aspartate receptor (dsDNA/NMDAR), anti-P and anti-neuronal surface $\mathrm{P}$ antigen (NSPA). Baseline and last follow-up: activity index (SLEDAI2K), systemic damage index (SDI). Statistics: multivariate analyses.

Results At baseline: mean age (37.0 years; SD 12.7), disease duration (7.2 years; SD 8.2), SLEDAI-2K score (7.4; SD 6.0) and SDI score (0.8; SD 1.3). Major depression (23\%), cognitive deficit (18\%) and both (3.4\%). APS (9\%). Antimalarials (84\%) and cytotoxics (36\%). Anti-dsDNA (49\%), dsDNA/ NMDAR (19\%), anti-P (12\%) and anti-NSPA (5\%). After a mean follow-up of 4.8 (SD 2.0) years, $11.1 \%$ acquired new damage. Final SDI score was 1.0 (SD 1.6). In multivariate analysis baseline SDI, SLEDAI-2K and cytotoxics associated 
Abstract 93 Table 1 Baseline lupus factors influencing damage accrual in 99 lupus patients. Regression analysis

\begin{tabular}{|c|c|c|c|}
\hline & OR & $\begin{array}{l}95 \% \text { confidence } \\
\text { limits }\end{array}$ & $p$ value \\
\hline \multicolumn{4}{|l|}{ New damage accrual } \\
\hline Intercept & 0.085 & - & 0.024 \\
\hline Cytotoxics use (yes) & 12.64 & $0.88-182.47$ & 0.062 \\
\hline SLEDAI-2K & 1.130 & $0.996-1.28$ & 0.057 \\
\hline Anti-NSPA positivity & 17.604 & $1.13-273.52$ & 0.040 \\
\hline \multicolumn{4}{|c|}{ New damage accrual excluding anti-NSPA } \\
\hline Intercept & 0.20 & - & $<0.001$ \\
\hline Cytotoxics use (yes) & 11.866 & $1.39-101.53$ & 0.024 \\
\hline SLEDAI-2K & 1.149 & $1.03-1.29$ & 0.016 \\
\hline
\end{tabular}

with final damage whereas SLEDAI-2K and cytotoxics associated with accrual damage. Models including anti-NSPA showed its impact on final and accrual damage. Cognitive deficit, depression and other autoantibodies were not damage predictors.

Conclusions Our findings support that disease activity and cytotoxic use are relevant lupus damage predictors. Cognitive dysfunction and depression do not contribute to damage accrual as may fluctuate in lupus patients. A potential influence of anti-NSPA antibodies on damage accrual is proposed.

Funding Source(s): FONDECYT grant \# 1160513 to LM and CONICYT Basal grant \# AFB170005 to AG

\section{DEVELOPMENT OF SYSTEMIC LUPUS ERYTHEMATOSUS SYMPTOMS IS ASSOCIATED WITH CIGARETTE SMOKING}

${ }^{1}$ Karoline Lerang*, 'Torhild Garen, 'Inge-Margrethe Gilboe, ${ }^{2}$ Jan Tore Gran. 'Oslo University Hospital, Rikshospitalet; ${ }^{2}$ University of Oslo

\subsection{6/lupus-2019-Ism.94}

Background Smoking is associated with the development of aCCP positive rheumatoid arthritis. The association between smoking and SLE is more uncertain. 1) We wanted to compare SLE patients smoking habits before symptom development with population controls.

Methods 306 SLE patients fulfilling ACR classification criteria from Oslo area were sent a questionnaire in 2010 regarding smoking. Antibody profiles were collected from medical records.

Year of first symptom was defined by the patient, or if the doctors time of the first SLE symptom was earlier, this year was used. Juvenile SLE was defined by start of symptom before 16 years of age. One pack year was 20 cigarettes daily in one year.

The proportion smokers in the Norwegian population were found online 2) and every SLE patient was matched with population controls for the year of the first symptom, age (number not available for every years of age, but 10 year groups) and gender.

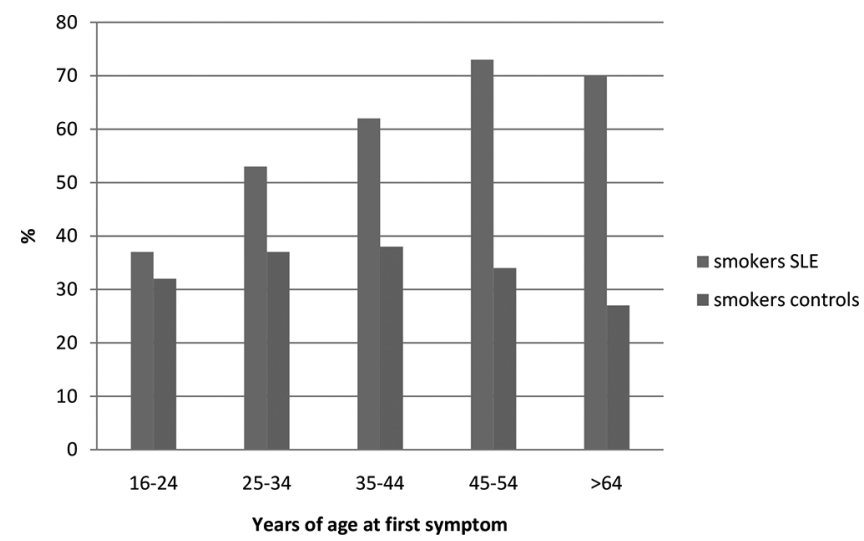

Abstract 94 Figure 1 The proportion of adult SLE patient smoking at the time of their first SLE symptom

Results 255/306 (80\%) SLE patients responded to the questionnaire. The mean age in 2010 was 48 years old (SD 15, min $18 \max 98)$ There were 39 patients with juvenile SLE whereof $64 \%$ were never smokers and $4 / 39$ (10\%) were smoking at the time of the 1 . symptom.

Among adult SLE, 116 (42\%) were never smokers and 83 (35\%) were previous smokers. In year 201053 (22\%) SLE patients were still smoking vs $20 \%$ in the control population (ns).

109/216 (50\%) adult SLE patients were smoking at the time of the first SLE symptom vs $35 \%$ in the matched control population $(\mathrm{p}<0,05)$, figure 1 . SLE patients had been smoking for a mean of 14 years when first symptom appeared and the mean pack year before first symptom were 10 (SD 11). The mean pack year after first symptom were 1 (SD 5).

Ever smokers did not have more dsDNA, aSm or aRNP antibodies, but they had a tendency to more discoid lupus (17\% vs $8 \%$ ).

Conclusions Even though prevalent SLE patients do not smoke more than their population controls, SLE patients are more often smokers at the time of the first symptom, especially in the higher age groups. This might indicate smoking as an environmental risk factor for developing SLE. However, smoking is tightly related to several other socioeconomic and cultural factors which must be explored in a prospective manner. Funding Source(s): None

\section{THE PERFORMANCE OF A RENAL ACTIVITY INDEX IN LUPUS NEPHRITIS IN INDUCTION THERAPY}

${ }^{1}$ Najla Aljaberi ${ }^{*},{ }^{2}$ Theresa Hennard, ${ }^{1}$ Arjun Mathur, ${ }^{2}$ Hermine I Brunner. ${ }^{1}$ Cincinnati Children's Hospital; ${ }^{2}$ Cincinnati Children's Hospital Medical Center

\subsection{6/lupus-2019-Ism.95}

Background Renal involvement in systemic lupus erythematosus (SLE) is associated with high morbidity and mortality. Current standard tools to monitor lupus nephritis (LN) are suboptimal compared to the invasive renal biopsy. The renal activity index in lupus (RAIL) was developed using 6 urinary biomarkers to reflect disease activity. In children this tool was $92 \%$ accurate in identifying active LN. We aim to study the changes in this score in relation to induction treatment in $\mathrm{LN}$. 